\title{
Optimistic chordal coloring: a coalescing heuristic for SSA form programs
}

\author{
Philip Brisk • Ajay K. Verma • Paolo Ienne
}

Received: 21 July 2008 / Accepted: 27 October 2008 / Published online: 11 November 2008

(C) Springer Science+Business Media, LLC 2008

\begin{abstract}
The interference graph for a procedure in Static Single Assignment (SSA) Form is chordal. Since the $k$-colorability problem can be solved in polynomial-time for chordal graphs, this result has generated interest in SSA-based heuristics for spilling and coalescing. Since copies can be folded during SSA construction, instances of the coalescing problem under SSA have fewer affinities than traditional methods. This paper presents Optimistic Chordal Coloring (OCC), a coalescing heuristic for chordal graphs. OCC was evaluated on interference graphs from embedded/multimedia benchmarks: in all cases, OCC found the optimal solution, and ran, on average, $2.30 \times$ faster than Iterated Register Coalescing.
\end{abstract}

Keywords Algorithms · Performance $\cdot$ Coalescing $\cdot$ Chordal graphs

\section{Introduction}

Register allocation is one of the most widely studied NP-Complete problems in computer science. Register allocation is typically broken down into two sub-problems: spilling and coalescing, both of which are NP-Complete [3-5, 19, 31, 33]. Spilling is the problem of partitioning all of the variables that are live at each point in the program between registers and memory. Coalescing is the problem of assigning variables to registers such that: (1) no variables whose lifetimes overlap are assigned to the same register; and (2) the minimum (weighted) number of register-to-register copy instructions remain in the program (or, equivalently, the maximum number of copies are removed).

For many years, register allocation and other related storage assignment problems have been modeled using graph coloring, another well-known NP-Complete problem [12, 13, 17, 18, 29], or its inverse, clique partitioning [39]. Although graph coloring is NP-Complete in the general case, there are many classes of graphs for which polynomial-time coloring

This paper is an extension of a paper that appeared at CASES 2007 [10].

P. Brisk $(\bowtie) \cdot$ A.K. Verma · P. Ienne

Swiss Federal Institute of Technology, Lausanne (EPFL), Switzerland

e-mail: philip.brisk@epfl.ch 
solutions are known. Two such classes of particular importance are chordal graphs [20] and interval graphs [26].

Recently, there has been interest in performing register allocation using intermediate representations using Static Single Assignment (SSA) Form; pruned SSA Form [14] is assumed throughout the paper. In particular, several research groups have recently proven that the interference graph for a program in SSA Form belongs to the class of chordal graphs [2, 8, 24]; Brisk and Sarrafzadeh [9] also proved that the interference graph for a program in Static Single Information (SSI) Form, an extension of SSA Form, belongs to the class of interval graphs. Unfortunately, both spilling and coalescing remain NP-Complete for chordal and interval graphs $[4,5]$; spilling is NP-Complete for straight-line code with no control flow [19].

That being said, SSA Form does have some advantages that could be useful for register allocation. First and foremost, the problem of spill-free register allocation can be solved optimally in polynomial-time: i.e., if the target architecture has $k$ registers, then a minimum coloring of the interference graph determines whether spilling is necessary; if spilling is unnecessary, then a solution to the coalescing problem must be found that eliminates as many copy instructions as possible. A second benefit is that all of the copy operations in the program can be eliminated during the conversion to SSA Form [6]. Copies are only inserted during the translation out of SSA Form to eliminate $\varphi$-functions: conditional parallel copy operations that are an integral part of SSA Form [16]. The number of copies to be eliminated via coalescing in SSA Form is significantly less than traditional register allocation.

\subsection{Contribution}

This paper contributes a novel heuristic for the coalescing problem for SSA Form programs whose interference graph is chordal. The heuristic is called Optimistic Chordal Coloring $(O C C)$, and it ensures that a legal coloring is found while attempting to minimize the (weighted) number of copy operations that remain in the program after coloring the interference graph. The worst-case time complexity of OCC is $O\left(|V|^{2}\right)$.

OCC was tested on a set of interference graphs generated from a set of Mediabench [30] and MiBench [23] applications and compared against the well-established Iterated Register Coalescing (IRC) heuristic [21] and an optimal formulation (OPT) of the coalescing problem as an integer linear program (ILP) [22]. In all of our test cases, OCC found the optimal solution to the problem, while running more than twice as fast as IRC, on average.

\subsection{The coalescing problem}

Consider variables, $u$ and $v$, connected by a copy operation $v \leftarrow u$. If both $u$ and $v$ are assigned to the same register, $r$, then the copy will become $r \leftarrow r$-an identity operation that does not change the state of the processor - a NOP which can be eliminated.

Let $V$ be the set of variables in the program. Two variables interfere if their lifetimes overlap. Let $E \subseteq V \times V$ be the set of interference edges, i.e. $e=\left(v_{1}, v_{2}\right) \in E$ if $v_{1}$ and $v_{2}$ interfere. Let $A \subseteq V \times V-E$ be the set of affinity edges, i.e., $a=\left(v_{1}, v_{2}\right) \in A$ if and only if $v_{1}$ and $v_{2}$ do not interfere and there is a copy operation $v_{1} \leftarrow v_{2}$ or $v_{2} \leftarrow v_{1}$ in the program. If runtime profiling information is available, and the copy is known to execute $w$ times, then the affinity edge is given a weight, denoted $w(a)=w(u, v)$, i.e. $a=\left(v_{1}, v_{2}, w\right)$.

The graph $G=(V, E, A)$ is called an interference graph. It can be constructed as described in the textbook by Cooper and Torczon [15]. A $k$-coloring is a function color : $V \rightarrow$ $\{1,2, \ldots, k\}$. A $k$-coloring is legal if for every interference edge $\left(v_{1}, v_{2}\right) \in E, \operatorname{color}\left(v_{1}\right) \neq$ 
color $\left(v_{2}\right)$; otherwise, it is illegal. An affinity edge $a=\left(v_{1}, v_{2}\right)$ is satisfied by a $k$-coloring color if color $\left(v_{1}\right)=\operatorname{color}\left(v_{2}\right)$. An unsatisfied affinity edge requires the insertion of a copy operation $v_{1} \leftarrow v_{2}$, or $v_{2} \leftarrow v_{1}$; a satisfied affinity edge eliminates the copy by assigning $v_{1}$ and $v_{2}$ to the same register.

In general, the problem of determining whether or not a graph is $k$-colorable is NPComplete; however, in the case of the coalescing problem, we are given an interference graph that is known to be $k$-colorable, where $k$ is the number of registers in the target architecture. The goal of the coalescing problem is to find a $k$-coloring of the interference graph that maximizes the sum of the weights of the satisfied affinity edges.

In SSA Form, all copies can be folded; however, affinities are introduced by $\varphi$-functions, an integral part of the SSA Form. To understand this paper, the reader does not need to understand the details of SSA Form or how $\varphi$-functions are instantiated; it suffices to understand the concept of an affinity edge.

The details of the translation out of SSA Form can be found in the paper by Hack and Goos [24]. Similar to their model, we assume that swap instructions are available for SSA elimination, when needed. The details are beyond the scope of this work.

\subsection{Paper organization}

Section 2 introduces preliminary notation and concepts; Sect. 3 summarizes related work on coalescing; Sect. 4 presents the OCC heuristic; Sect. 5 presents the experimental evaluation and results; Sect. 6 concludes the paper.

\subsection{Statement of extension of prior work}

The version of OCC presented here is an extension of prior work [10]. Material that is unique to this paper includes: the example interference graphs taken from Mediabench and MiBench applications; the discussion of illegal pseudo-coalescing (Sect. 4.4.2); the BMCS2 method to compute a PEO (Sect. 4.3); the full-blown color assignment and propagation method (Sect. 4.5.2); complexity analyses of OCC (Sect. 4.6); new experimental results based on a new implementation of OCC (Sect. 5).

\section{Preliminaries}

\subsection{Graph notation}

Let $G=(V, E, A, w)$ be an interference graph as introduced in Sect. 1.1. $\chi(G)$ is defined to be the chromatic number of $G$, i.e., the smallest value $k$ for which there is a legal $k$-coloring for $G$. For vertex $v$, let: $N(v)$ be the set of interference neighbors of $v ; N_{A}(v)$ be the set of affinity neighbors of $v ; R_{A}(v)$ be the set vertices reachable from $v$ by affinity edges; and $C_{A}(v)$ be a set of vertices with which $v$ has been pseudo-coalesced (see Sect. 4.2). Let $S$ be a set of vertices. Then:

$$
N(S)=\bigcup_{v \in S} N(v)-S
$$




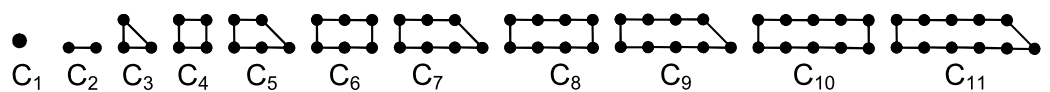

Fig. $1 k$-cycles $C_{1}, \ldots, C_{11}$

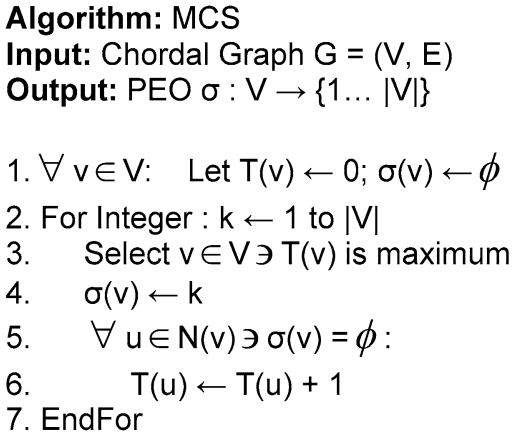

(a)
Algorithm: Chordal Color

Input: Chordal Graph $\mathrm{G}=(\mathrm{V}, \mathrm{E}), \mathrm{PEO} \sigma$

Output: Color Assignment $f: V \rightarrow\left\{1 \ldots \chi_{G}\right\}$

1. For Integer : $\mathrm{i} \leftarrow 1$ to $|\mathrm{V}|$ in PEO order

2. Let $\mathrm{c}$ be the smallest color not assigned

3. $\operatorname{color}\left(\mathrm{v}_{\mathrm{i}}\right) \leftarrow \mathrm{c}$ to a vertex in $\mathrm{N}^{\mathrm{i}}\left(\mathrm{v}_{\mathrm{i}}\right)$

4. EndFor

Fig. 2 Pseudocode for MCS [38] (a) and optimal chordal color assignment [20] (b)

\subsection{Chordal graphs}

A $k$-cycle is the graph $C_{k}=\left(U_{k}, E_{k}\right)$, where $U_{k}=\left\{v_{0}, v_{1}, \ldots, v_{k-1}\right\}$ and $E_{k}=\left\{\left(v_{i}\right.\right.$, $\left.\left.v_{(i+1) \bmod k}\right) \mid 0 \leq i \leq k-1\right\}$. Figure 1 lists the $k$-cycles $C_{1}, \ldots, C_{11}$. Graph $G=(V, E)$ is chordal if it has no subgraph isomorphic to a $C_{k}$ for $k \geq 4$; other equivalent definitions of chordal also exist. The interference graph of an SSA Form procedure is provably chordal [2, $8,24]$.

An Elimination $\operatorname{Order}(E O)$ is a one-to-one and onto function $\sigma: V \rightarrow\{1,2, \ldots,|V|\}$; given an EO, vertices are renamed so that $\sigma\left(v_{i}\right)=i$. Let $V_{i}=\left\{v_{1}, v_{2}, \ldots, v_{i}\right\}$ and $G_{i}=$ $\left(V_{i}, E_{i}\right)$ be the subgraph of $G$ induced by $V_{i}$. For vertex $v_{i}: N^{i}\left(v_{i}\right)=N\left(v_{i}\right) \cap V_{i}, N_{A}^{i}\left(v_{i}\right)=$ $N_{A}\left(v_{i}\right) \cap V_{i}$, and $C_{A}^{i}\left(v_{i}\right)=C_{A}\left(v_{i}\right) \cap V_{i}$.

Vertex $v$ is simplicial if $N(v)$ forms a clique. A Perfect Elimination Order (PEO) is an EO where $v_{i}$ is simplicial in $G_{i}$ for $1 \leq i \leq|V|$. Graph $G$ is chordal if and only if $G$ has a PEO. A PEO can be computed in $O(|V|+|E|)$ time by an algorithm called Maximum Cardinality Search (MCS) [38] shown in Fig. 2(a). Given a PEO, $G$ is colored optimally in $O(|V|+|E|)$ time using an algorithm shown in Fig. 2(b) [20]. Colors are assigned to vertices in PEO order. Then color $\left(v_{i}\right)$ is the smallest color not assigned to a vertex in $N^{i}\left(v_{i}\right)$; optimality ensues because $N^{i}\left(v_{i}\right)$ is a clique.

\section{Related work}

In graph coloring register allocation, coalescing refers to the process of merging vertices in the interference graph, often to eliminate copy operations via register assignment. Given an affinity edge $(u, v) \in A$, coalescing $u$ and $v$ ensures that they receive the same color. Let $u v$ be the resulting vertex. Then:

$$
\begin{aligned}
N(u v) & =N(u) \cup N(v), \\
N_{A}(u v) & =\left[N_{A}(u) \cup N_{A}(v)\right]-N(u v) .
\end{aligned}
$$




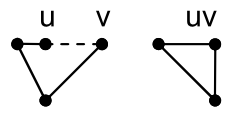

(a)

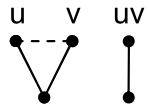

(b)

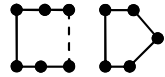

(c)

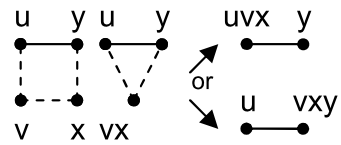

(d)

Fig. 3 Coalescing can increase the degree of the merged vertex and the chromatic number of the graph (a); it can also decrease the degree of a vertex adjacent to the two vertices that have been merged (b); and it can cause a chordal graph to become non-chordal, while increasing the chromatic number of the graph (c); in some cases, it is impossible to coalesce every affinity edge (d)

If affinity edges $(u, x)$ and $(v, x)$, exist then $w(u v, x)=w(u, x)+w(v, x)$.

Coalescing has both positive and negative side effects [32, 40]. In Fig. 3(a), $|N(u)|=$ $|N(v)|=1$ and $\chi_{G}=2$ before coalescing, and afterwards $|N(u v)|=2$ and $\chi_{G}=3$. In Fig. 3(b), $|N(u)|=|N(v)|=2$ before coalescing, and $|N(u v)|=1$ afterwards. In Fig. 3(c) coalescing causes a chordal graph to become non-chordal and increases $\chi_{G}$ from 2 to 3 . Figure 3(d) shows that it is generally impossible to coalesce all affinity edges.

Let $k$ be the number of registers in the target processor and assume that $\chi_{G} \leq k$. Three variants of the coalescing problem are NP-Complete [5]:

Aggressive coalescing $[12,13]$ tries to satisfy as many affinity edges as possible without constraining $\chi_{G}$; in register allocation, aggressive coalescing can increase the number of spills. Aggressive coalescing can also minimize the number of copy instructions required to eliminate $\varphi$-functions during translation out of SSA Form. Heuristics for aggressive coalescing have been proposed by Sreedhar et al. [37], Budimlic et al. [11], Rastello et al. [35] and Boissinot el al. [1].

Conservative coalescing [7, 21, 25, 27] attempts to find a legal $k$-coloring of $G$ such that $\chi_{G} \leq k$ and as many affinity edges as possible are satisfied.

Optimistic coalescing [32] begins with an aggressively coalesced interference graph $G$, and performs a de-coalescing phase that tries to minimize the number of affinity edges decoalesced while ensuring that $\chi_{G} \leq k$. In their study, Park and Moon [32] found that optimistic coalescing eliminated more copies than conservative coalescing.

Previous heuristics for conservative and optimistic coalescing are based on vertex merging. In SSA Form, the fact that the interference graph is chordal yields a stronger guarantee of $k$-colorability than traditional coalescing methods, because merging vertices may not preserve the chordal graph property; the color assignment procedure guarantees a legal coloring because it uses the same correctness invariant as Gavril's [20] chordal coloring algorithm. Pseudo-coalescing, introduced in Sect. 4.2, mimics the behavior of coalescing but without merging vertices. Thus, the OCC heuristic has stronger conservative guarantees than conservative coalescing, while retaining the benefits of optimistic coalescing in terms of solution quality.

\section{Optimistic chordal coloring}

The Optimistic Chordal Coloring (OCC) heuristic consists of six sequential steps, described in Sects. 4.1-4.5:

(1) Simplify: Remove vertices of low degree incident on no affinity edges.

(2) Pseudo-coalesce: Find (independent) sets of affinity-connected vertices.

(3) PEO: Compute a PEO; favor vertices incident on high-weight affinity edges. 
(4) Color Assignment: Assign colors to vertices in PEO order maintaining Gavril's invariant; color assignment is biased by the sets of pseudo-coalesced vertices.

(5) Refinement: Attempt to improve the solution via local improvement.

(6) Unsimplify: Assign colors to the vertices removed in Step (1).

The key points of the heuristic are as follows:

- Steps (3) and (4) exploit the fact that chordal graphs are $k$-colorable.

- Step (2) is cognizant of the fact that traditional coalescing heuristics often find good solutions. Step (4) tries to assign the same color to all vertices that have been pseudo-coalesced with one another; but it cannot guarantee that such a coloring is found.

- Steps (1), (3), and (6) exploit the observation that only vertices incident on affinity edges contribute to the objective value of the solution; all other vertices must be colored to ensure legality; if possible, deferring the assignment of colors to these vertices tends to yield better conservative solutions.

Sections 4.1-4.5 describe the 6 steps in detail; the complexity of OCC is analyzed in Sect. 4.6. The interference graph $G=(V, E, A)$ is assumed to be chordal throughout.

\subsection{Steps (1)/(6): simplify/unsimplify}

Let $v \in V$ be a vertex with $|N(v)|<k$. Then the subgraph of $G$ induced by $V-\{v\}$ is $k$-colorable if and only if $G$ is $k$-colorable [28]; it is also chordal. $v$ is called a simplifiable vertex. Simplify repeatedly removes each simplifiable vertex $v$ with $\left|N_{A}(v)\right|=0$ from $G$ and pushes $v$ onto a stack. Steps (2)-(5) then compute a $k$-coloring of the resulting chordal interference graph. Unsimplify repeatedly pops a vertex $v$ from the stack and reattaches it to $G$. When processing $v,|N(v)| \leq k-1$, so a color is available for $v$.

Some variation of simplification has been used during register allocation dating back to the first graph coloring allocator by Chaitin et al. [13] and Chaitin [12]. Our approach to simplification is the same as that of Hack and Grund [22].

Condition $\left|N_{A}(v)\right|=0$ ensures that removing $v$ cannot degrade the solution quality. Let $u \in N(v)$. If $\left|N_{A}(u)\right|>0$, then color $(u)$ influences the solution quality; assigning a color to $v$ before $u$ can only constraint the spectrum of colors available for $u$ when a color is chosen; thus, removing $v$ is beneficial at best and benign at worst. If $|N(u)|=k$ and $\left|N_{A}(u)\right|=0$, then the removal of $v$ renders $u$ simplifiable.

In Steps (2)-(5), all vertices in $V$ are unsimplifiable or are incident on at least one affinity edge.

\subsection{Step (2): pseudo-coalescing}

The purpose of pseudo-coalescing is to find a set of affinity-connected vertices; these sets are used to guide the color assignment phase in Step (4). Unlike traditional coalescing methods, these vertices are not merged. $C_{A}(v)$ is defined to be the set of vertices with which $v$ is pseudo-coalesced. Section 4.2.1 presents Optimistic Pseudo-coalescing [9], an adaptation of Park and Moon's [32] heuristic for aggressive coalescing. Section 4.2.2 introduces Illegal Pseudo-coalescing, in which $C_{A}(v)$ is defined to be the set of vertices reachable from $v$ via affinity edges.

\subsubsection{Optimistic pseudo-coalescing}

Initially, let $C_{A}(v)=\{v\}$ for each vertex $v$ with $\left|N_{A}(v)\right|>0$; the invariant that $C_{A}(v)$ is an independent set is maintained throughout. First, the set $A$ of affinity edges is sorted in de- 


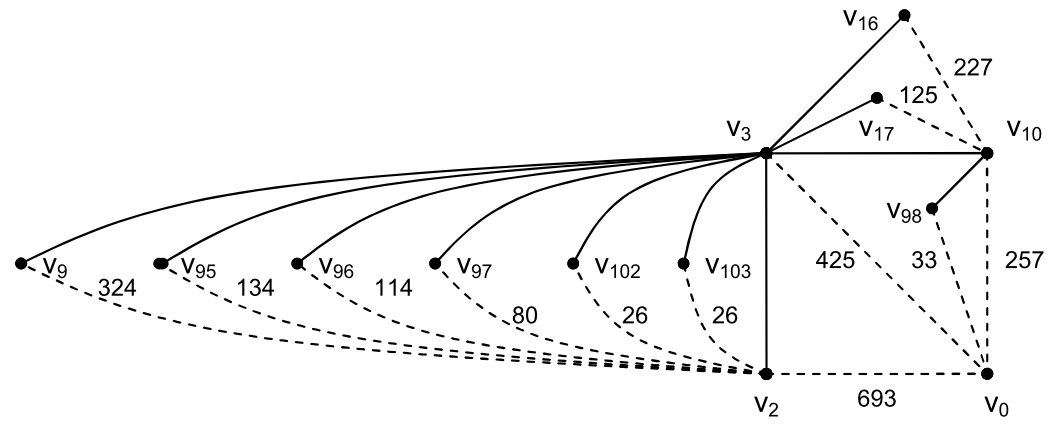

Fig. 4 An interference graph fragment taken from the frame_ME function in the mpeg2enc benchmark. After optimistic pseudo-coalescing, $C_{A}\left(v_{0}\right)=\left\{v_{0}, v_{2}, v_{9}, v_{10}, v_{16}, v_{17}, v_{95}, v_{96}, v_{97}, v_{102}, v_{103}\right\}$, $C_{A}\left(v_{3}\right)=\left\{v_{3}\right\}$, and $C_{A}\left(v_{98}\right)=\left\{v_{98}\right\}$, which leaves affinity edges $\left(v_{0}, v_{3}\right)$ and $\left(v_{0}, v_{98}\right)$ unsatisfied

scending order of weight; affinity edges are processed in sorted order. Consider affinity edge $\left(v_{1}, v_{2}, w\right)$. If $C_{A}\left(v_{1}\right)=C_{A}\left(v_{2}\right)$, then $v_{1}$ and $v_{2}$ are already pseudo-coalesced; otherwise, $v_{1}$ and $v_{2}$ are pseudo-coalesced if and only if $C_{A}\left(v_{1}\right) \cup C_{A}\left(v_{2}\right)$ is an independent set; it suffices to check that $C_{A}\left(v_{1}\right) \cap N\left(C_{A}\left(v_{2}\right)\right)$ (or, equivalently, $\left.N\left(C_{A}\left(v_{1}\right)\right) \cap C_{A}\left(v_{2}\right)\right)$ is empty. Sorting the affinity edges in advance favors pseudo-coalescing of high-weight affinity edges over lower-weight affinity edges.

Figure 4 shows a fragment of the interference graph for the procedure frame_ME taken from the $M P E G$-2 encoder benchmark [30]; many vertices are not shown, for clarity. Pseudo-coalescing affinity edges in sorted order, yields independent sets: $C_{A}\left(v_{0}\right)=$ $\left\{v_{0}, v_{2}, v_{9}, v_{10}, v_{16}, v_{17}, v_{95}, v_{96}, v_{97}, v_{102}, v_{103}\right\}, C_{A}\left(v_{3}\right)=\left\{v_{3}\right\}$, and $C_{A}\left(v_{98}\right)=\left\{v_{98}\right\}$; affinity edges $\left(v_{0}, v_{3}\right)$ and $\left(v_{0}, v_{98}\right)$ are unsatisfied. This solution is optimal.

\subsubsection{Illegal pseudo-coalescing}

The use of one NP-Complete problem to solve another is a poor strategy; thus, the use of aggressive coalescing, which is NP-Complete in its own right [5], within a heuristic to solve conservative coalescing, is a poor strategy: even if aggressive coalescing is solved optimally, it does not guarantee an optimal solution to conservative coalescing. Thus, the time spent on finding an aggressive solution would be better spent searching for a conservative solution directly. To this end, the illegal pseudo-coalescing strategy replaces the optimistic strategy with a much more efficient computation, sidestepping this issue completely.

Let $R_{A}(v)$ be the set of vertices reachable from $v$ by affinity edges. Under illegal pseudocoalescing: $C_{A}(v)=R_{A}(v)$ for each vertex $v$. When illegal coalescing is performed, $C_{A}(v)$ may not be an independent set; thus, illegal pseudo-coalescing does not attempt to solve the aggressive coalescing problem; for example $C_{A}\left(v_{0}\right)$ in Fig. 4 would contain every vertex in $G$, including those that interfere. This is not, however, problematic: there is no actual requirement that $C_{A}\left(v_{0}\right)$ be an independent set. Color assignment and propagation, in Step (4), is given leeway to de- and re-coalesce vertices in an adaptive fashion when one color cannot be assigned to all of the vertices in $C_{A}(v)$.

The details of illegal pseudo-coalescing cannot be understood without first understanding the color assignment heuristic, and are therefore delayed until Sect. 4.4.6. 


\subsection{Step (3): compute PEO}

A graph is chordal if and only if it has a PEO; in fact, it may have many distinct PEOs. Gavril's [20] algorithm can use any PEO to compute a $k$-coloring. The quality of coalescing solutions produced by OCC, however, which account for affinity edges, is highly dependent on the PEO used. The ideal PEO, intuitively, assigns colors to vertices incident on highweight affinity edges as early as possible during its execution.

A Biased Maximum Cardinality Search (BMCS) [9] makes the following modification to the MCS algorithm. Referring to Fig. 2(a), let $M$ be the set of vertices $v$ such that $T(v)$ is maximum (line 3). For vertex $m \in M$, let $W^{*}(m)$ be the sum of the weights of the affinity edges incident on $m$. When there is a choice between multiple vertices with maximum $T$ values, $W^{*}$ is used as a tiebreaker, and the vertex with the maximum $w^{*}$ value is chosen. No other modifications are required.

A second alternative, BMCS-2, is a minor modification to BMCS. For vertex $m \in M$, let $W_{2}^{*}(m)$ be the sum of the weights of the affinity edges incident on $m$ such that the other vertex incident on the affinity edge precedes $m$ in the PEO. When there is a choice between multiple vertices with maximal $T$-values, $W_{2}^{*}$ is used as a tiebreaker; $W^{*}$ is used as a second tiebreaker if multiple vertices remain.

In most cases, BMCS and BMCS-2 find the same solution; however, we identified one case where BMCS-2 does better. This example, once again, can only be understood in the context of Step (4), and is delayed until Sect. 4.4.7.

\subsection{Step (4): color assignment and propagation}

Gavril's [20] algorithm for optimal chordal coloring does not account for affinity edges and their weights when assigning colors. OCC's color assignment and propagation is an adaptation of Gavril's algorithm to perform better coalescing; it borrows the following correctness invariant: a legal $k$-coloring is maintained for the induced subgraph $G_{i}=\left(V_{i}, E_{i}\right)$ when vertex $v_{i}$ is assigned a color. OCC's method, however, is completely different because it must account for affinity edge weights.

Section 4.4.1 describes a simplified version of the color assignment heuristic. Section 4.4.2 introduces the process of de- and re-pseudo-coalescing, which are unique contributions of this paper; examples are shown in Sects. 4.4.3 and 4.4.4. Section 4.4.5 describes the full heuristic in detail. Lastly, Sects. 4.4.6 and 4.4.7 present the examples alluded to in Sects. 4.2 and 4.3; these examples illustrate situations where illegal pseudo-coalescing outperforms optimistic pseudo-coalescing and where BMCS-2 outperforms BMCS.

\subsubsection{Simplified color assignment heuristic}

Let $C_{A}\left(v_{i}\right)$ be the set of vertices with which $v_{i}$ is pseudo-coalesced. OCC makes every effort to assign the same color to all of the vertices in $C_{A}\left(v_{i}\right)$; unfortunately, this is not always possible. Let $v_{i}$ be the first vertex in $C_{A}\left(v_{i}\right)$ to receive a color $c=\operatorname{color}\left(v_{i}\right)$. There must be an affinity edge $a=\left(v_{i}, v_{j}, w\right), j>i$, such that $v_{j} \in C_{A}\left(v_{i}\right)$; otherwise, there would be no incentive, from the perspective of satisfying affinity edges, to assign the same color to $v_{i}$ and the other vertices in $C_{A}\left(v_{i}\right) . v_{i}$ receives a color before $v_{j}$ since $j>i$.

When processing $v_{j}$, the ideal color to select would be $c$, since this choice would satisfy $a$. This is similar to biased coloring [7]. Unfortunately, there may be a vertex $v_{l}, i<l<j$, that interferes with $v_{j}$ and has $\operatorname{color}\left(v_{l}\right)=c$. Thus, after assigning $c$ to $v_{i}$, we also wish to bias the choice of color assigned to $v_{l}$ away from $c$. To do this, OCC optimistically 


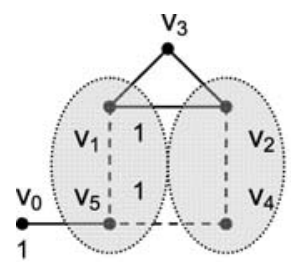

(a)

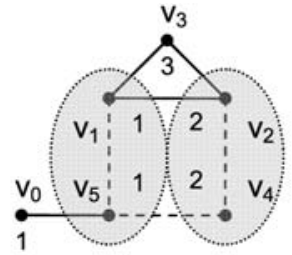

(b)

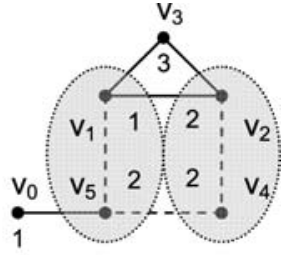

(c)

Fig. 5 Illustration of color assignment and propagation: a pre-assigned color cannot be confirmed for $v_{5}$. $v_{1}$ and $v_{5}$ are pseudo-coalesced, as are $v_{2}$ and $v_{4}$. Color 1 is assigned to $v_{0}$ and $v_{1}$, and then propagated to $v_{5}$ (a); color 2 is assigned to $v_{2}$ and propagated to $v_{4}$; color 3 is assigned to $v_{3}$ (b). $v_{4}$ confirms color 2 ; $v_{5}$ cannot confirm color 1 due to the interference with $v_{0}$; given a choice between colors 2 and $3, v_{5}$ selects color 2 due to the affinity with $v_{4}(\mathbf{d})$

propagates color $c$ to all vertices in $C_{A}\left(v_{i}\right)$, including $v_{j}$, when $c$ is assigned to $v_{i}$. OCC may change the color when $v_{j}$ is processed; this does not violate the invariant since $v_{j} \notin V_{i}$. The choice of color assigned to $v_{l}$ is biased away from $c$. If no other colors are available for $v_{l}$ when $v_{l}$ is processed, then $c$ will be chosen for $v_{l}$; the fact that interfering vertices $v_{l}$ and $v_{j}$ have the same color do not violate the invariant since $v_{j} \notin V_{l} ; v_{j}$, in this case, will not receive color $c$.

Let free_colors be the set of colors available for $v_{i}$, as used in traditional chordal color assignment; i.e.: free_colors contains the colors not assigned to vertices in $N^{i}\left(v_{i}\right)$. Let optimistic_free_colors be the set of colors not assigned to vertices in $N\left(v_{i}\right)$. optimistic_free_ colors accounts for colors that have been propagated to vertices occurring after $v_{i}$ in the PEO. If possible, a color from optimistic_free_colors is selected for $v_{i}$; if optimistic_free_colors is empty, then a color from free_colors is selected instead. Since the interference graph is chordal, free_colors is non-empty.

When $c$ is propagated to $v_{i}$, it is assumed that $c$ is preferred when choosing $\operatorname{color}\left(v_{i}\right)$. If $c \in$ free_colors, then $c$ is assigned to $v_{i}$; this process is called confirmation; otherwise $v_{i}$ cannot confirm its pre-assigned color $c$. If there is an affinity edge $\left(v_{i}, v_{k}, w\right)$, and $\operatorname{color}\left(v_{k}\right)=c^{\prime}$, then $c^{\prime}$ can be chosen for $v_{i}$ as long as $c^{\prime} \in$ free_colors.

Figure 5 shows an example, where optimistic pseudo-coalescing is used. Initially, $v_{1}$ and $v_{5}$ are optimistically pseudo-coalesced, as are $v_{2}$ and $v_{4} . v_{0}$ and $v_{1}$ are assigned color 1 , which is then propagated from $v_{1}$ to $v_{5} . v_{2}, v_{3}$, and $v_{4}$ are assigned colors 2,3 , and 2. $v_{5}$, however, cannot confirm $1 ; 2$ is chosen instead to satisfy affinity edge $\left(v_{4}, v_{5}\right)$.

\subsubsection{De- and re-pseudo-coalescing}

If $v_{i}$ cannot confirm $c$, then another color should be chosen for $v_{i}$. Although not explicitly stated above, this effectively de-pseudo-coalesces $v_{i}$ from the other vertices in $C_{A}\left(v_{i}\right)$. Assigning color 2 to $v_{5}$ in Fig. 5(c) de-pseudo-coalesces $v_{5}$ from $v_{1}$; furthermore, the choice to assign color 2 to $v_{5}$ effectively re-pseudo-coalesces $v_{5}$ with $v_{4}$ and $v_{2}$.

Let $v_{i}$ be the first vertex in $C_{A}\left(v_{i}\right)$ to be assigned a color, $c$; then $c$ is propagated to the other vertices in $C_{A}\left(v_{i}\right)$. If later, $c$ cannot be confirmed for some vertex $v_{j} \in C_{A}\left(v_{i}\right)$, then $v_{j}$ is de-pseudo-coalesced from $C_{A}\left(v_{i}\right)$, yielding a new set, $L_{A}\left(v_{j}\right)=C_{A}\left(v_{i}\right)-\left\{v_{j}\right\}$. We then attempt to re-pseudo-coalesce $v_{j}$ with a new set of pseudo-coalesced vertices: $C_{A}\left(v_{k}\right)$ for some other vertex $v_{k} \notin L_{A}\left(v_{j}\right)$, where adding $v_{j}$ to $C_{A}\left(v_{k}\right)$ satisfies at least one affinity. It may also be possible to attract some affinity neighbors of $v_{i}$ that belong to $L_{A}\left(v_{j}\right)$ into $C_{A}\left(v_{k}\right)$; details of this process are presented in the following examples. 


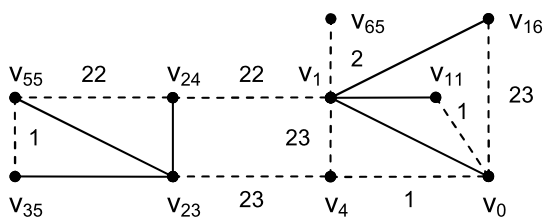

Fig. 6 Part of the interference graph for the sym_decrypt function, used to illustrate re-coalescing. The optimal solution satisfies all affinity edges except $\left(v_{1}, v_{4}, 23\right)$; leaving $\left(v_{1}, v_{24}, 22\right)$ and $\left(v_{0}, v_{4}, 1\right)$ unsatisfied is an equivalent optimal solution

\subsubsection{Example 1: sym_decrypt}

Figure 6 shows part of the interference graph of the sym_decrypt function of the pegwit benchmark [30]. Assume that illegal pseudo-coalescing is used: $C_{A}\left(v_{0}\right)$ contains all of the vertices. First, color 1 is assigned to $v_{0}$ and propagated to the vertices in $C_{A}\left(v_{0}\right)$. Since $v_{1}$ interferes with $v_{0}, v_{1}$ is de-pseudo-coalesced from $C_{A}\left(v_{0}\right)$ and color 2 is assigned to $v_{1}$.

Now, consider vertex $v_{65}$; the only affinity edge incident on $v_{65}$ is $\left(v_{1}, v_{65}, 2\right)$; there is no benefit to keeping color 1 assigned to $v_{65}$, since this color can no longer satisfy this affinity. Instead, $v_{1}$ is able to de-pseudo coalesce $v_{65}$ from $C_{A}\left(v_{0}\right)$ and re-pseudo-coalesce $v_{65}$ with $C_{A}\left(v_{1}\right)$.

Continuing with the example, $v_{4}$ and $v_{24}$ remain in $C_{A}\left(v_{0}\right)$. $v_{4}$ is incident on two affinity edges $\left(v_{0}, v_{4}, 1\right)$ and $\left(v_{4}, v_{23}, 23\right)$, in addition to $\left(v_{1}, v_{4}, 23\right)$. Keeping 1 as $v_{4}$ 's color satisfies the first two affinity edges, whose weight totals 24 ; changing $v_{4}$ 's color to 2 would satisfy the third affinity edge whose weight is 23 ; therefore, $v_{4}$ keeps its color and remains in $C_{A}\left(v_{0}\right)$.

In PEO order, $v_{4}, v_{11}, v_{16}$ and $v_{23}$ all confirm pre-assigned color $1 . v_{24}$ cannot confirm color 1 since it interferes with $v_{23}$. Therefore, $v_{24}$ de-pseudo-coalesces itself from $C_{A}\left(v_{0}\right)$; the only other set of pseudo-coalesced vertices incident on $v_{24}$ is $C_{A}\left(v_{1}\right)$; therefore, $v_{24}$ is repseudo-coalesced with $C_{A}\left(v_{1}\right), v_{24}$ then invites $v_{55}$ to de-pseudo-coalesce from $C_{A}\left(v_{0}\right)$ and re-pseudo-coalesce with $C_{A}\left(v_{1}\right)$. Retaining $v_{55}$ 's membership in $C_{A}\left(v_{0}\right)$ yields a net benefit of 1 , due to affinity edge $\left(v_{35}, v_{55}, 1\right)$, while de-pseudo-coalescing and re-pseudo-coalescing with $C_{A}\left(v_{1}\right)$ yields a net benefit of 22 due to affinity edge $\left(v_{24}, v_{55}, 22\right)$; the latter option is preferable, so $v_{55}$ is removed from $C_{A}\left(v_{0}\right)$ and inserted into $C_{A}\left(v_{1}\right)$; by similar reasoning, $v_{35}$ is removed from $C_{A}\left(v_{0}\right)$ and inserted into $C_{A}\left(v_{1}\right)$ as well.

As the heuristic proceeds, color 2 is confirmed for $v_{35}, v_{55}$, and $v_{65}$, in order. This yields an optimal solution, wherein the only unsatisfied affinity edge is $\left(v_{1}, v_{4}, 23\right)$; another optimal solution leaves affinity edges $\left(v_{0}, v_{4}, 1\right)$ and $\left(v_{1}, v_{24}, 22\right)$ unsatisfied.

\subsubsection{Example 2: RPE_grid_positioning}

A fragment of the interference graph fragment for function $R P E \_$grid_positioning from the gsm benchmark [30] is shown in Fig. 7. Assume that illegal pseudo-coalescing is used: $C_{A}\left(v_{2}\right)$ contains all of the vertices in the interference graph.

First, color 1 is assigned to vertex $v_{2}$, and is propagated to all other vertices in the graph. $v_{13}$ and $v_{22}$ confirm color $1 ; v_{31}$ takes color 2 instead, due to an interference with $v_{22}$. Vertices $v_{53}$ and $v_{59}$ are successfully attracted into $C_{A}\left(v_{22}\right)$, so $C_{A}\left(v_{2}\right)=$ $\left\{v_{2}, v_{13}, v_{22}, v_{46}, v_{47}, v_{52}, v_{57}\right\}$ and $C_{A}\left(v_{31}\right)=\left\{v_{31}, v_{53}, v_{59}\right\} . v_{46}$ then confirms color $1 ; v_{47}$ cannot confirm color 1 due to interference edge $\left(v_{46}, v_{47}\right) ; v_{47}$ is de-pseudo-coalesced from $C_{A}\left(v_{2}\right)$, and successfully attracts $v_{57}$ as well. At this point, $C_{A}\left(v_{2}\right)=\left\{v_{2}, v_{13}, v_{22}, v_{46}, v_{52}\right\}$, $C_{A}\left(v_{31}\right)=\left\{v_{31}, v_{53}, v_{59}\right\}$ and $C_{A}\left(v_{47}\right)=\left\{v_{47}, v_{57}\right\} . v_{52}, v_{53}, v_{57}$, and $v_{59}$ then confirm their 
Fig. 7 Part of the interference graph for the

RPE_grid_positioning function used to illustrate de- and re-coalescing. The optimal solution leaves affinity edges $\left(v_{22}, v_{57}, 9161\right)$ and $\left(v_{22}, v_{53}, 167\right)$ unsatisfied

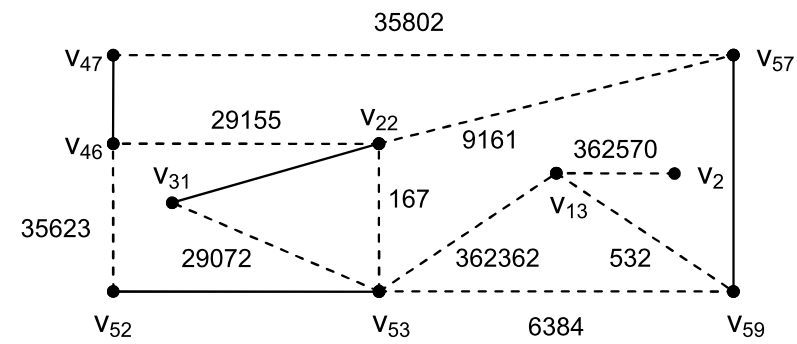

colors in order. This yields two unsatisfied affinity edges: $\left(v_{22}, v_{53}, 167\right)$ and $\left(v_{22}, v_{57}, 9161\right)$ : the optimal solution.

\subsubsection{Detailed description of color assignment and propagation}

Assume that vertex $v_{i}$ cannot confirm its pre-assigned color, so it must be de-pseudocoalesced from $C_{A}\left(v_{i}\right)$; recall that $L_{A}\left(v_{i}\right)=C_{A}\left(v_{i}\right)-\left\{v_{i}\right\}$. Let $v_{j} \notin L_{A}\left(v_{i}\right)$ be a vertex that is affinity-adjacent to $v_{i}$. Here, we entertain the possibility of adding $v_{i}$ to $C_{A}\left(v_{j}\right) . v_{i}$ cannot be added to $C_{A}\left(v_{j}\right)$ if $v_{i}$ interferes with at least on vertex in $C_{A}\left(v_{j}\right)$, or if $v_{i}$ has an interference neighbor $v_{k}$ such that $\operatorname{color}\left(v_{k}\right)=\operatorname{color}\left(v_{j}\right)$; otherwise, it is possible to add $v_{i}$ to $C_{A}\left(v_{j}\right)$. There may be multiple sets of pseudo-coalesced vertices to which $v_{i}$ is affinityadjacent; in this case, we must select the best one in terms of satisfying affinity edges.

The gain associated with pseudo-coalescing $v_{i}$ with $C_{A}\left(v_{j}\right)$, under the assumption that doing so is legal, is as follows:

$$
\operatorname{gain}\left(v_{i}, C_{A}\left(v_{j}\right)\right)=\sum_{\substack{v_{k} \in C_{A}\left(v_{j}\right) \\\left(v_{i}, v_{k}\right) \in A}} w\left(v_{i}, v_{k}\right)
$$

$v_{i}$ is added to the set of pseudo-coalesced vertices $C_{A}\left(v_{j}\right)$ such that $\operatorname{gain}\left(v_{i}, C_{A}\left(v_{j}\right)\right)$ is maximal. If there is no set $C_{A}\left(v_{j}\right)$ with positive aggregate gain, then $v_{i}$ is inserted into a singleton set, i.e.: $C_{A}\left(v_{i}\right)=\left\{v_{i}\right\}$. If the vertices in the chosen set have been colored, then $v_{i}$ is assigned that color; if they have not been colored, then an appropriate color is assigned to $v_{i}$ and that color is propagated to all of the vertices in $C_{A}\left(v_{i}\right)$.

Now, we should entertain the possibility of de-pseudo-coalesecing affinity neighbors of $v_{i}$ from their current sets and re-pseudo-coalescing them with $C_{A}\left(v_{i}\right)$, as was done in the case of vertices $v_{1}$ and $v_{24}$ in Fig. 6, and $v_{31}$ and $v_{47}$ in Fig. 7; this is done only if doing so increases the number of satisfied affinity edges.

The process itself is a breadth-first search. Initially, each affinity neighbor $u$ of $v_{i}$ is inserted into an empty queue, denoted $Q$. At every step, a vertex is dequeued from $Q$. A test, described below, determines whether $u$ is de-pseudo-coalesced from its current set and re-pseudo-coalesced with $C_{A}\left(v_{i}\right)$, or not. If $u$ passes the test and is brought into $C_{A}\left(v_{i}\right)$, then each affinity neighbor of $u$ that is neither enqueued nor belongs to $C_{A}\left(v_{i}\right)$ already is enqueued. The process repeats until $Q$ is empty. This process may bring many of the vertices in $L_{A}\left(v_{i}\right)$ into the new $C_{A}\left(v_{i}\right)$.

Without loss of generality, let us dequeue vertex $u$. If $u$ interferes with any vertex in $C_{A}\left(v_{i}\right)$, or if $u$ is assigned the same color as any vertex in $N\left(C_{A}\left(v_{i}\right)\right)$, then $u$ cannot be pseudo-coalesced with $C_{A}\left(v_{i}\right)$. Otherwise, it is perfectly legal to de-pseudo-coalesce $u$ from $C_{A}(u)$ and re-pseudo-coalesce $u$ with $C_{A}\left(v_{i}\right)$; however, this should only be done if doing so improves the objective function. 
Let component_gain $(u)$ be the gain associated with leaving $u$ in $C_{A}(u)$ and component_ $\operatorname{gain}\left(v_{i}\right)$ be the gain associated with bringing $u$ into $C_{A}\left(v_{i}\right)$ :

$$
\begin{aligned}
& \text { component_gain }(u)=\sum_{x \in N_{A}(u) \cap C_{A}(u)} w(u, x), \\
& \text { component_gain }\left(v_{i}\right)=\sum_{y \in N_{A}(u) \cap C_{A}\left(v_{i}\right)} w(u, y) .
\end{aligned}
$$

If component_gain $(u)>$ component_gain $\left(v_{i}\right)$, then it is beneficial to remove $v_{i}$ from $C_{A}\left(v_{i}\right)$ and insert $v_{i}$ into $C_{A}(u)$; otherwise, $v_{i}$ remains in $C_{A}\left(v_{i}\right)$.

For example, refer back to processing vertex $v_{53}$ in Fig. 7 , which is attracted to $C_{A}\left(v_{31}\right)$. component_gain $\left(v_{53}\right)=w\left(v_{22}, v_{53}\right)+w\left(v_{53}, v_{59}\right)=167+6384=6551$; meanwhile, component_gain $\left(v_{31}\right)=w\left(v_{31}, v_{53}\right)=29072>$ component_gain $\left(v_{53}\right)$. Therefore, $v_{53}$ de-pseudo-coalesces with $C_{A}\left(v_{53}\right)$ and re-pseudo-coalesces with $C_{A}\left(v_{31}\right)$.

Likewise, when $v_{47}$ attracts $v_{57}$ in Fig. 7, component_gain $\left(v_{57}\right)=w\left(v_{22}, v_{57}\right)=9161$, while component_gain $\left(v_{47}\right)=w\left(v_{47}, v_{57}\right)=35802$. Therefore, $v_{57}$ de-pseudo-coalesces with $C_{A}\left(v_{57}\right)$ and re-pseudo-coalesces with $C_{A}\left(v_{47}\right)$.

\subsubsection{Example 3: susan_smoothing}

A fragment of the interference graph of the procedure susan_smoothing, taken from the susan benchmark [23], will be used to illustrate the advantage of illegal over optimistic pseudocoalescing; it is shown in Fig. 8. The optimal solution leaves affinity edges $\left(v_{0}, v_{31}\right)$ and $\left(v_{56}, v_{60}\right)$ unsatisfied. Since many affinity edges have the same weight, optimistic pseudocoalescing may produce different results, depending on the order in which the edges are processed. The worst possible solution is $C_{A}\left(v_{0}\right)=\left\{v_{0}, v_{31}, v_{33}, v_{35}, v_{53}, v_{56}, v_{60}\right\}$, which leaves 4 unsatisfied affinity edges: $\left(v_{29}, v_{31}, 1\right),\left(v_{30}, v_{31}, 1\right),\left(v_{58}, v_{60}, 1\right)$, and $\left(v_{59}, v_{60}, 1\right)$. In this case, color 1 is assigned to $v_{0}$ and then propagated to the remaining vertices in $C_{A}\left(v_{0}\right)$; color 1 is then confirmed when each vertex is processed during color assignment.

Under illegal pseudo-coalescing, $C_{A}\left(v_{0}\right)$ initially contains all of the vertices in Fig. 8. $v_{0}$ is assigned color $1 ; v_{29}$ and $v_{30}$, which both interfere with $v_{0}$ are assigned color 2 . When $v_{29}$ is assigned color 2, it de-pseudo-coalesces from $C_{A}\left(v_{0}\right)$ and tries to attract $v_{31}$ into $C_{A}\left(v_{29}\right)$; the attraction fails, because $v_{0}$ and $v_{30}$, the two other affinity neighbors of $v_{31}$, belong to $C_{A}\left(v_{0}\right)$. Assigning color 2 to $v_{30}$ also de-pseudo-coalesces $v_{30}$ from $C_{A}\left(v_{0}\right)$. Thus, at this point, $C_{A}\left(v_{29}\right)=\left\{v_{29}\right\}$ and $C_{A}\left(v_{30}\right)=\left\{v_{30}\right\} . v_{30}$ successfully attracts $v_{31}$ into $C_{A}\left(v_{30}\right)$, since $v_{31}$ has two affinity neighbors with color $2\left(v_{29}\right.$ and $\left.v_{30}\right)$ and one with color $1\left(v_{0}\right) ; v_{31}$ then successfully attracts $v_{29}$ into $C_{A}\left(v_{31}\right)=C_{A}\left(v_{30}\right)$. The same thing happens on the left-handside of Fig. 8 with vertices $v_{58}, v_{59}$, and $v_{60}$, yielding an optimal solution.

Fig. 8 Part of the interference graph for the susan_smoothing function, used to illustrated re-coalescing

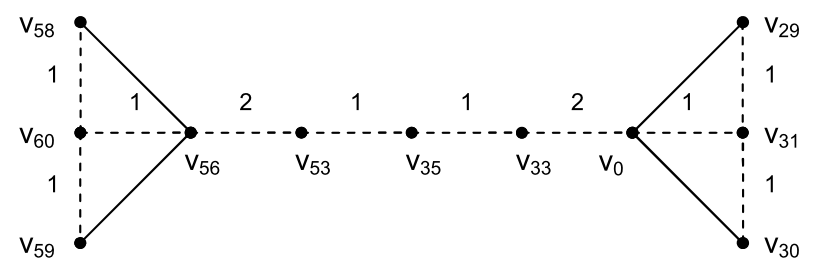


Fig. 9 Part of the interference graph for the jpeg_fill_bit_buffer function

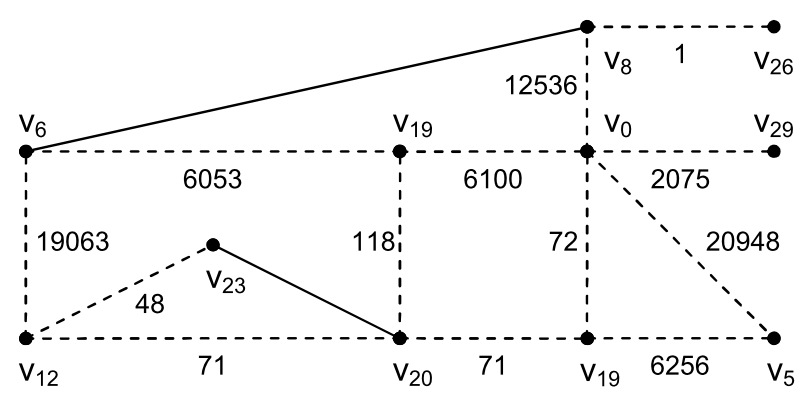

\subsubsection{Example 4: jpeg_fill_bit_buffer}

Figure 9 shows a fragment of the interference graph of the jpeg_fill_bit_buffer function from the jpeg_6a benchmark [30]. The optimal solution leaves affinity edges $\left(v_{6}, v_{14}, 6053\right)$ and $\left(v_{12}, v_{20}, 71\right)$ unsatisfied. Vertices are listed in their BMCS ordering; this example will show that BMCS-2 yields a better solution.

The difference between the PEOs generated by the BMCS and BMCS-2 heuristic involves vertex $v_{6}$ and $v_{8} . v_{0}$ is the first vertex selected; $v_{1}, \ldots, v_{5}$ are not shown in Fig. 9. In the traditional BMCS, $W^{*}\left(v_{8}\right)=12357>W^{*}\left(v_{6}\right)=25116$, so $v_{6}$ precedes $v_{8}$; in BMCS-2, $W_{2}^{*}\left(v_{6}\right)=0<W_{2}^{*}\left(v_{8}\right)=12356, v_{8}$ would precede $v_{6}$ in the PEO. We assume that illegal pseudo-coalescing is used in both cases.

Consider the vertices in BMCS order. First, vertex $v_{0}$ receives color 1 , which is propagated to the other vertices; $v_{5}$ and $v_{6}$ confirm color 1 . Due to the interference edge $\left(v_{6}, v_{8}\right), v_{8}$ does not confirm color 1 and choses color 2 instead, which leaves affinity edge $\left(v_{0}, v_{8}, 12356\right)$ unsatisfied: this is sub-optimal. $v_{8}$ is unable to attract $v_{0}$ to $C_{A}\left(v_{8}\right)$, due to the weights of the other three affinity edges incident on $v_{0}$, which sum to 29195: all of the vertices incident on three edges still belong to $C_{A}\left(v_{0}\right)$. In the PEO produced by BMCS-2, $\sigma\left(v_{8}\right)=6, \sigma\left(v_{6}\right)=8$; the other vertices keep the same order as the BMCS. $v_{8}$ confirms color 1 , while $v_{6}$ is forced to accept color 2 . $v_{6}$ then successfully attracts $v_{12}$ and $v_{23}$ into $C_{A}\left(v_{6}\right)$; the remaining vertices then confirm their colors and the optimal solution is found.

\subsection{Refinement}

OCC guarantees a legal coloring but does not guarantee optimality in terms of the number of affinities satisfied. Two refinement techniques introduced in the next two subsections, can be used to try to locally improve the coalescing solution of OCC.

\subsubsection{Local refinement}

Local refinement processes each affinity edge $a=(u, v, w)$ where $u$ and $v$ are assigned different colors. If swapping the colors assigned to $u$ and $v$ causes an illegal coloring, then $a$ is discarded. If the swap is legal, then the swap is accepted if it improves the solution quality; otherwise, the swap is suppressed. After deciding to accept or reject the swap, the next affinity edge is processed.

\subsubsection{Aggressive refinement}

Like its local counterpart, aggressive refinement processes affinity edges one-by-one, only refining those that are not satisfied; in this case, the refinement process is much more complicated and has a greater time complexity per miscolored affinity edge. 


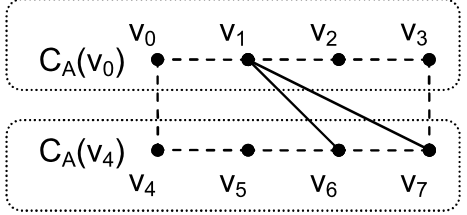

(a)

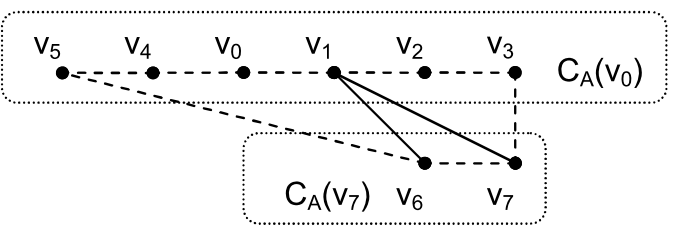

(b)

Fig. 10 Illustration of aggressive refinement. Two sets of pseudo-coalesced vertices are shown (a); after aggressive refinement, vertices $v_{4}$ and $v_{5}$ can be de-pseudo-coalesced from $C_{A}\left(v_{7}\right)$ and re-pseudo-coalesced with $C_{A}\left(v_{0}\right)$ because they do not interfere with any vertices in $C_{A}\left(v_{0}\right) ; v_{6}$ and $v_{7}$ remain in $C_{A}\left(v_{7}\right)$ after pseudo-coalescing because they interfere with $v_{1} \in C_{A}\left(v_{0}\right)$

Let $a=(u, v)$ be an unsatisfied affinity edge. The intuitive explanation for the aggressive refinement step, illustrated in Fig. 10, is as follows: we consider the possibility of decoalescing all of the vertices in $C_{A}(v)$ that do not interfere with any vertices in $C_{A}(u)$, and re-psuedo-coalescing all of these vertices into $C_{A}(u)$. This is done only if it improves the objective function; it may also be necessary to find a new color to assign to the vertices in $C_{A}(u)$ after this transformation, in order to ensure legality.

The first step is to compute a set, free_colors, which contains a spectrum of colors which we may choose to assign to $u$ and $v$ during refinement. Ideally, we will find a new color that can be assigned to both $u$ and $v$, thereby satisfying $a$, that can also be assigned to some of the vertices in $C_{A}(u)$ and $C_{A}(v)$, while ensuring a legal coloring and improving the objective function. Initially, free_colors $=\{1,2, \ldots, k\}$. For each vertex $n \in N\left(C_{A}(u)\right) \cup N\left(C_{A}(v)\right)$, color $(n)$ is removed from free_colors. If free_colors becomes empty, we discard $a$ and move on to the next unsatisfied affinity edge; otherwise, we proceed with $a$.free_colors is no longer needed after this point. It simply evaluates the feasibility of finding a color assignment that satisfies $a$ given the colors assigned to the remainder of the interference graph.

Let $T_{u, v}=N\left(C_{A}(u)\right) \cap C_{A}(v)$; these are the sets of vertices belonging to $C_{A}(v)$ that cannot possibly be re-pseudo-coalesced with $C_{A}(u)$ due to interferences, e.g.: if $v_{0}=u$ and $v_{4}=v$ in Fig. 10(a), then $T_{u, v}=\left\{v_{6}, v_{7}\right\}$ due to the interference edges. Aggressive refinement tries to de-pseudo-coalesce all of the vertices belonging to $C_{A}(v)-T_{u, v}$ and re-pseudo-coalesce them with $C_{A}(u)$ instead.

Let $t$ be a vertex in $T_{u, v}$. The interfering vertex affinity cost of $t$, denoted $I(t)$, effectively measures the force with which the vertices in $T_{u, v}$ hold onto the remaining vertices in $C_{A}(v)$. $I(t)$ is the sum of the weights of the affinity edges between $t$ and its affinity neighbors in $C_{A}(v)$, i.e.:

$$
I(t)=\sum_{x \in N_{A}(t) \cap C_{A}(v)} w(t, x) .
$$

The aggregate interfering vertex affinity cost, denoted $I^{*}$, is the sum of the interfering vertex affinity costs over all vertices in $T_{u, v}$ :

$$
I^{*}=\sum_{t \in T_{u, v}} I(t)
$$

$I^{*}$ effectively measures the estimated benefit of doing nothing, i.e.: leaving the vertices in $C_{A}(v)-T_{u, v}$ pseudo-coalesced with $T_{u, v}$. 
The counterpart to $I^{*}$, which measures the strength with which vertices in $C_{A}(u)$ attract vertices belonging to $C_{A}(v)-T_{u, v}$ is called the merged component affinity cost, and is denoted $M^{*}$. Let $z \in C_{A}(v)-T_{u, v}$. The merged component affinity cost associated with $z$, denoted $M(z)$, is computed as follows:

$$
M(z)=\sum_{x \in N_{A}(z) \cap C_{A}(u)} w(x, z) .
$$

$M^{*}$ is then computed as follows:

$$
M^{*}=\sum_{z \in C_{A}(v)-T_{u, v}} M(z) .
$$

If $M^{*}>I^{*}$, then it is beneficial to de-pseudo-coalesce the vertices in $C_{A}(v)-T_{u, v}$ from $C_{A}(v)$ and re-pseudo-coalesce them with $C_{A}(u)$. Let $C_{A}^{*}(u)=C_{A}(u) \cup\left(C_{A}(v)-T_{u, v}\right)$ be a new name given to $C_{A}(u)$ after the re-pseudo-coalescing. In general, we may not be able to simply assign the same color used for $C_{A}(u)$ to the vertices in $C_{A}(v)-T_{u, v}$ due to interferences. Thus, we entertain the possibility of recoloring all of the vertices in $C_{A}^{*}(u)$ and $T_{u, v}$ in order to ensure legality. If $v \in C_{A}^{*}(u)$, then a new color must be found for the vertices in $C_{A}(v) \cap T_{u, v}$ as well. This is done using free_colors arrays, as described previously, for both $C_{A}^{*}(u)$ and $T_{u, v}$ : if a free color is found for the vertices in $C_{A}^{*}(u)$ and for $T_{u, v}$, then the aggressive refinement has been successful for affinity edge $a$; otherwise, it is rejected since it cannot find a legal coloring.

Aggressive refinement is not commutative with respect to both $u$ and $v$. If aggressive refinement fails, the process can be repeated for $a$ with the roles of $u$ and $v$ reversed.

If aggressive refinement fails, the process can be repeated for affinity edge $a^{\prime}=(v, u)$, which is the same affinity edge, but with the roles of $u$ and $v$ reversed.

\subsubsection{Example of aggressive refinement}

Figure 11 shows a fragment of the interference graph for the procedure get_dht from the jpeg_djpeg benchmark. The table on the right-hand-side of Fig. 11 shows the color assignment before and after aggressive refinement. Initially, four affinity edges are not satisfied: $\left(v_{8}, v_{11}, 348\right),\left(v_{29}, v_{32}, 64\right),\left(v_{46}, v_{48}, 8\right)$ and $\left(v_{39}, v_{48}, 4\right)$; after aggressive refinement, the optimal solution leaves three affinity edges unsatisfied: $\left(v_{8}, v_{11}, 348\right),\left(v_{29}, v_{32}, 64\right)$, and $\left(v_{43}, v_{48}, 4\right)$.

During aggressive refinement, unsatisfied affinity edges are processed in descending order of weight. Aggressive refinement fails for the first three affinity edges, but triggers recoloring when processing the unsatisfied affinity edge $\left(v_{39}, v_{48}, 4\right)$.

Let $u=v_{39}$ and $v=v_{48} . C_{A}(u)=\left\{v_{0}, v_{8}, v_{9}, v_{25}, v_{29}, v_{30}, v_{39}, v_{46}, v_{49}, v_{55}, v_{58}, v_{61}\right\}$ and $C_{A}(v)=\left\{v_{43}, v_{48}\right\} ; T_{u, v}=\left\{v_{43}\right\}$ due to the interference edge $\left(v_{39}, v_{43}\right) . I^{*}=4$, which is the weight of affinity edge $\left(v_{39}, v_{48}, 4\right)$; there is no other affinity edge incident on $v_{39}$. $M^{*}=12$, due to affinity edges $\left(v_{39}, v_{48}, 4\right)$ and $\left(v_{46}, v_{48}, 8\right)$.

All of the vertices in $C_{A}(u)$ are initially assigned color 0 ; this color is unavailable for $v_{48}$ : although not shown in Fig. 11, $v_{48}$ interferes with another vertex whose color is 0 . The search for a free color for the set $C_{A}^{*}(u)=C_{A}(u) \cup\left\{v_{48}\right\}$ finds 10 as a free color. $v_{43}$ retains its originally assigned color, 9. 


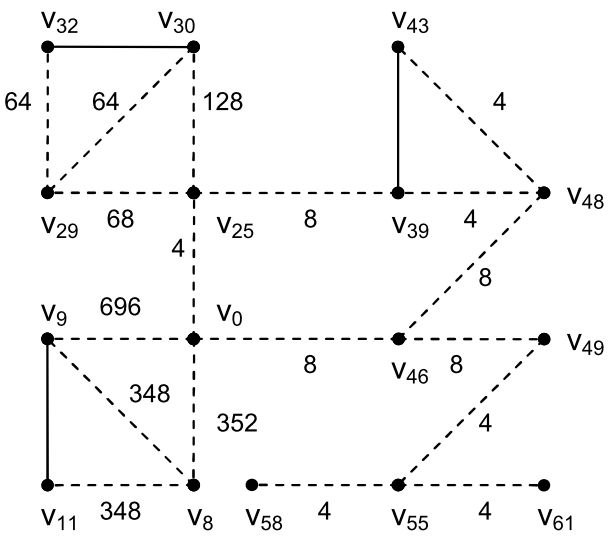

\begin{tabular}{|c|c|c|}
\hline Vertex & Before & After \\
\hline $\mathrm{v}_{0}$ & 0 & 10 \\
\hline$V_{8}$ & 0 & 10 \\
\hline$v_{9}$ & 0 & 10 \\
\hline$v_{11}$ & 7 & 7 \\
\hline$v_{25}$ & 0 & 10 \\
\hline$v_{29}$ & 0 & 10 \\
\hline$v_{30}$ & 0 & 10 \\
\hline$v_{32}$ & 8 & 8 \\
\hline$v_{39}$ & 0 & 10 \\
\hline$v_{43}$ & 9 & 9 \\
\hline$v_{46}$ & 0 & 10 \\
\hline$V_{48}$ & 9 & 10 \\
\hline$V_{49}$ & 0 & 10 \\
\hline$V_{55}$ & 0 & 10 \\
\hline$v_{58}$ & 0 & 10 \\
\hline$v_{61}$ & 0 & 10 \\
\hline
\end{tabular}

Fig. 11 Illustration of aggressive refinement on a portion of the interference graph for the procedure get_dht in the jpeg_djpeg benchmark. The color assigned to each vertex is shown before and after aggressive refinement; miscolored affinity edge $(39,48,4)$ triggers the re-coloring

\subsection{Time complexity}

\subsubsection{Time complexity of simplification}

The time complexity of simplification is $O(|V|+|E|)$ : each vertex must be examined once: when a vertex is removed, the degree of its neighbors is reduced, following which the neighbor can be tested for simplifiability.

\subsubsection{Time complexity of optimistic pseudo-coalescing}

The time complexity of optimistic pseudo-coalescing is $O[|A|(\log |A|+|V|+|E|)]$. The time complexity of sorting the affinity edges is $O(|A| \log |A|)$, the time complexity of a comparison-based sort; alternatively, if the maximum affinity edge weight is $W$, then Counting Sort, which has a time complexity of $O(|A|+W)$, can be used [36]. Each affinity edge must be processed, and the time complexity of enumerating the neighborhood $N(S)$ of each set of pseudo-coalesced vertices $S$ is $O(|V|+|E|)$ : the time complexity of a depth- or breadth-first search; this justifies the $O[|A|(|V|+|E|)]$ term.

\subsubsection{Time complexity of illegal pseudo-coalescing}

The time complexity of illegal pseudo-coalescing is $O(|V|+|A|)$ : the $R_{A}$ sets are found by a breadth/depth-first search through the affinity edges of the interference graph.

\subsubsection{Time complexity of color assignment and propagation}

The time complexity of Gavril's chordal coloring algorithm is $O(|V|+|E|)$. Let $P$ represent the complexity of the color propagation step for each vertex. Thus, the overall time complexity is $O(|V| P+|E|)$; the complexity of the $P$ is discussed next.

During color assignment and propagation, $u$ is the vertex that has been removed from the queue; we must process each affinity neighbor $v$ of $u$ to determine whether or not $v$ can be added to $C_{A}(u)$, which requires computing the component_gain terms. The time complexity 
of both of these steps is $T=O\left[\left|N\left(C_{A}(u)\right)\right|+\left|C_{A}(u)\right|+|N(v)|+\left|N_{A}(v)\right|\right]$, ignoring vertices that have already been enqueued; for the sake of simplicity, we assume that these complexity terms are the same for each vertex.

If $q$ is the total number of vertices that are enqueued during color propagation, so $P=$ $O(q T)$ and the overall complexity is $O(|V| q T+|E|)$. In actuality, there is a different $T$ term for each vertex, since the four components of the $T$ term vary from vertex-to-vertex (and may change dynamically as vertices are moved from one $C_{A}$ set to another). It follows that $q T=O(|V|)$, a loose bound, since at most $|V|$ vertices are processed each time color propagation is performed. At most $O(|A|)$ vertices will be uncovered during the breadth first search; however, $\left|N\left(C_{A}(u)\right)\right|+\left|N_{A}(v)\right|=O(|V|)$ in the worst case, since $\left|N\left(C_{A}(u)\right)\right|$ and $\left|N_{A}(v)\right|$ include their interference neighbors. Therefore, we report the overall complexity as $O\left(|V|^{2}+|E|\right)=O\left(|V|^{2}\right)$.

\subsubsection{Time complexity of local refinement}

The time complexity of processing an affinity edge $a=(u, v)$ is $O\left[|N(u)|+\left|N_{A}(u)\right|+\right.$ $\left.|N(v)|+\left|N_{A}(v)\right|\right]$. Local refinement examines the interference neighbors of $u$ and $v$ to determine the legality of the swap, and the affinity neighbors of $u$ and $v$ to incrementally compute objective function resulting from the swap. In the worst case, $O(A)$ affinity edges are processed. The complexity term for a single affinity edge is aggregated over all of the unsatisfied affinity edges. In the worst case, it becomes $O(|A||V|)$.

\subsubsection{Time complexity of aggressive refinement}

The time complexity of aggressive refinement is $O(|A||V|)$. In the worst case, $O(|A|)$ affinity edges must be processed, under the assumption that only a constant number of affinity edges are satisfied to begin with. For each unsatisfied affinity edge, $O(|V|)$ vertices must be processed in order to compute the $I$ and $M$ terms.

\subsubsection{Time complexity of unsimplify}

During unsimplification, each vertex $v$ is popped from the stack and reattached to the graph; it is given the smallest color not assigned to its neighbors. At most $|N(v)|$ neighbors are processed, and $|N(v)|+1$ colors are examined as possibilities for $v$; in aggregation, at most $|E|$ interferences edges in $G$ will be processed. Since at most $|V|$ vertices are on the stack to begin with, the time complexity is $O(|V|+|E|)$.

\subsubsection{Time complexity of $O C C$}

From Sects. 4.6.1-4.6.7, the time complexity of OCC is $O\left(|V|^{2}+|A||V|\right)$.

All of the copies in a procedure can be eliminated during SSA construction; thus, the only affinities that remain are those due to $\varphi$-functions. In practically all cases we have observed, $|A| \ll|V|$; under this assumption, the complexity simplifies to $O\left(|V|^{2}\right)$.

\section{Experimental results}

A set of embedded and multimedia benchmarks taken from the Mediabench [30] and MiBench [23] benchmark suites were selected for evaluation. The Machine SUIF compiler 
Table 1 Number of registers used for each benchmark

\begin{tabular}{llll}
\hline Benchmark & Registers & Benchmark & Registers \\
\hline adpcm_coder & 14 & jpeg_cjpeg & 18 \\
adpcm_decoder & 14 & jpeg_djpeg & 39 \\
blowfish & 14 & mpeg2dec & 21 \\
crc32 & 8 & mpeg2enc & 45 \\
dijkstra & 6 & patricia & 9 \\
FFT & 13 & pegwit & 13 \\
g721_decoder & 16 & sha & 10 \\
g721_encoder & 16 & susan & 20 \\
gsm & 16 & & \\
\hline
\end{tabular}

was used to compile an instrumented version of each application to collect profiling data (basic block execution frequencies) and to generate the interference graphs with affinity edges weighted based on the profiling information gathered during compilation. Each procedure was converted to SSA Form before generating its interference graph, which is provably chordal.

As our goal is to study the coalescing problem, we did not perform spilling. For each application let $G=\left\{G_{1}, \ldots, G_{n}\right\}$ be the set of interference graphs for each procedure; we targeted each application toward a RISC architecture with $R=\max \left\{\chi\left(G_{1}\right), \ldots, \chi\left(G_{n}\right)\right\}$ general purpose registers. $R$ is the minimum number of registers that ensure no variables must be spilled, except possibly across procedure calls. Since each procedure is in SSA Form, each $G_{i}$ is chordal, so $\chi\left(G_{i}\right)$ can be computed in polynomial-time [20].

Table 1 lists each benchmark and the number of registers in the corresponding target architecture. Each interference graph was colored 3 times: first using the OCC heuristic; second, using the well-established iterated coalescing (IRC) heuristic [21]; and third, using an optimal ILP formulation (OPT) [22].

Table 2 shows the number of copies dynamically executed for each benchmark, i.e.: the weighed sum of the unsatisfied affinity edges after coloring; the number of dynamically executed copies (based on the profiling information) is shown for OPT; the number of additional copies executed is shown for IRC and OCC. OCC was able to find the optimal coloring solution for each interference graph in the study; IRC was able to find optimal solutions for many, but not all of the benchmarks.

In Table 2, OCC was implemented with BMCS-2, illegal pseudo-coalescing, and both refinement stages (aggressive followed by local). To better understand the behavior of OCC, Table 3 presents results using four different configurations with refinement suppressed: OCC-BI (BMCS-2 + illegal pseudo-coalescing), OCC-BO (BMCS-2 + optimistic pseudocoalescing), OCC-MI (MCS + illegal pseudo-coalescing), and OCC-MO (MCS + optimistic pseudo-coalescing).

OCC-MI performed poorly: its results are substandard compared to the others, suggesting that illegal pseudo-coalescing is only useful in conjunction with a BMCS that tries to ensure that vertices incident on high-weight affinity edges are assigned colors as early as possible. OCC-BO and OCC-MO found the same results for all benchmarks: all but three benchmarks were solved optimally, and, across all benchmarks, only executed 7 copies in excess of the optimal solution.

Beginning with a set of optimistic pseudo-coalesced vertices, the lack of interferences between vertices that were pseudo-coalesced together minimized the amount of de- and re-pseudo-coalescing; the quality of the results, however, was dependent of the quality of 
Table 2 Number of dynamically executed copy instructions for each benchmark. - means that IRC/OCC found optimal solutions; $+X$ indicates that IRC found solutions that execute $X$ copies in excess of the optimal solution

Table 3 Number of dynamically executed copies for different configurations of the OCC heuristic

\begin{tabular}{lrll}
\hline Benchmark & \multicolumn{1}{l}{ OPT } & IRC & OCC \\
\hline adpcm_rawcaudio & 6995016 & - & - \\
adpcm_rawdaudio & 6995016 & - & - \\
blowfish & 0 & - & - \\
crc32 & 53322406 & - & - \\
dijkstra & 0 & - & - \\
fft & 8209 & - & - \\
g721_decode & 0 & - & - \\
g721_encode & 0 & - & - \\
gsm & 1909187 & +2990 & - \\
jpeg_cjpeg & 541326 & - & - \\
jpeg_djpeg & 272636 & +6283 & - \\
mpeg2dec & 2115 & - & - \\
mpeg2enc & 95197 & - & - \\
patricia & 1820 & - & - \\
pegwit & 48 & +24 & - \\
sha & 442 & - & - \\
susan & 2 & - & - \\
\hline
\end{tabular}

\begin{tabular}{lllll}
\hline Benchmark & OCC-BI & OCC-BO & OCC-MI & OCC-MO \\
\hline adpcm_rawcaudio & - & - & - & - \\
adpcm_rawdaudio & - & - & - & - \\
blowfish & - & - & - & - \\
crc32 & - & - & - & - \\
dijkstra & - & - & - & - \\
fft & - & - & - & - \\
g721_decode & - & - & - & - \\
g721_encode & - & - & - & - \\
gsm & - & - & +792005 & - \\
jpeg_cjpeg & - & - & - & - \\
jpeg_djpeg & +21 & +4 & +742 & +4 \\
mpeg2dec & - & - & - & - \\
mpeg2enc & - & - & +224 & - \\
patricia & - & - & +66971 & - \\
pegwit & - & +1 & +1 & +1 \\
sha & - & - & - & - \\
susan & - & +2 & +1 & +2 \\
\hline & & & &
\end{tabular}

the optimistic pseudo-coalescing; as there are known dependencies on the order in which affinity edges are pseudo-coalesced, this situation seems less than ideal, even if it the results were generally favorable.

OCC-BI found the optimal solution for all benchmarks except jpeg_djpeg, for which it executed 21 copies in excess of the optimal solution. With local, but not aggressive, re- 


\section{Refinement Overhead}

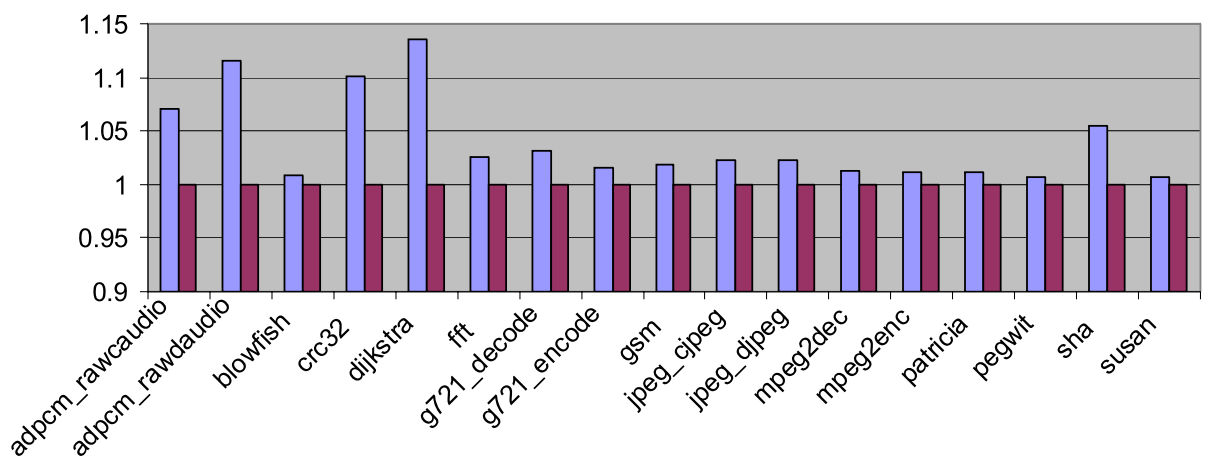

$\square O C C$ with refinement $\square O C C$ without refinement

Fig. 12 The overhead of performing refinement

finement the number of excess copies was reduced to +13 ; with aggressive, but not local, refinement, the number of excess copies was reduced to +8 ; with both types of refinement, the optimal solution was achieved.

Next, we measured the overhead of performing refinement. We colored each interference graph with the OCC-BI heuristic, with and without (both types of) refinement; for each measurement, each interference graph was colored 5000 times and the average runtime was taken. The results are shown in Fig. 12. The IRC and OCC heuristics were run on a Dell Latitude D810 laptop with an Intel Pentium M processor running at $2.0 \mathrm{GHz}$ with $1.0 \mathrm{~GB}$ of RAM; the operating system used was Fedora Core 5.

The overhead of refinement ranged from 1.00 (susan) to 1.14 (dijkstra). In general, the largest overheads were observed for the benchmarks with the smallest overall runtimes ( $a d$ pcm_rawcaudio, adpcm_rawdaudio, crc32, dijkstra, sha); these benchmarks all had very few interference graphs, all of which were colored quite quickly, thereby amplifying the impact of refinement. For the remaining benchmarks, the overhead of refinement was 1.03 or less.

The overhead of refinement is clearly tolerable for traditional "offline" compilation; however, for just-in-time (JIT) compilers, the overhead of refinement can only be justified by its savings in runtime execution. For OCC-MI, refinement saves a total of 21 dynamically executed instructions; this cannot be justified; as the compiler executes, the cost of saving/restoring caller/callee-save registers for the call to one (of the two) refinement function(s) alone will easily eclipse 21 instructions, let alone the runtime cost of performing the refinement.

It should also be noted that a JIT compiler is likely to use a faster register allocator, such as linear scan [34], or one of its variants, that sacrifices solution quality in order to reduce compile time; in particular, linear scan does not use an interference graph, and does not perform coalescing at all.

Table 4 compares the runtime of the OCC heuristic with the runtime of OPT and IRC. IRC and OCC were run using the same setup as described above. The experiments using OPT were performed by Sebastian Hack on a Dell Latitude D420 laptop with an Intel Core Duo $U 2500$ processor running at $1.2 \mathrm{GHz}$ and with $2.0 \mathrm{~GB}$ of RAM; the operating system was Ubuntu Feisty, and CPLEX 7.0 was used to solve the ILP. 
Table 4 Runtime (normalized to OCC) of OPT, IRC, and OCC

\begin{tabular}{lrll}
\hline Benchmark & OPT & IRC & OCC \\
\hline adpcm_rawcaudio & 904 & 3.47 & 1 \\
adpcm_rawdaudio & 2841 & 3.39 & 1 \\
blowfish & 3111 & 2.05 & 1 \\
crc32 & 139 & 1.28 & 1 \\
dijkstra & 188 & 1.12 & 1 \\
fft & 573 & 2.69 & 1 \\
g721_decode & 2394 & 3.36 & 1 \\
g721_encode & 3611 & 3.25 & 1 \\
gsm & 1403 & 2.21 & 1 \\
jpeg_cjpeg & 1659 & 2.13 & 1 \\
jpeg_djpeg & 10144 & 2.37 & 1 \\
mpeg2dec & 2661 & 2.03 & 1 \\
mpeg2enc & 2088 & 4.17 & 1 \\
patricia & 442 & 1.73 & 1 \\
pegwit & 140881 & 0.871 & 1 \\
sha & 670 & 2.88 & 1 \\
susan & 279256 & 0.105 & 1 \\
Average & 26645 & 2.30 & 1 \\
\hline
\end{tabular}

On average, the runtime of IRC was $2.3 \times$ greater than that of OCC. For two benchmarks, pegwit and susan, IRC was faster: significantly, in the case of susan. OPT, however, ran considerably slower than OCC: $139 \times$ to $279,256 \times$. This is to be expected, given that, absent a proof that $\mathrm{P}=\mathrm{NP}$, the optimal solution to NP-Complete problems can only be computed in exponential worst-case time. It should be noted that the implementation of the OCC heuristic used for these experiments is based on a different code base than an earlier version [9] that was published previously.

\section{Conclusion}

The optimistic chordal coloring (OCC) coalescing heuristic was introduced for chordal graphs, which arise from SSA Form programs. OCC is an extension of Gavril's [20] algorithm that computes a minimum coloring of a chordal graph. OCC maintains the same correctness invariant as Gavril's algorithm, which offers stronger conservative guarantees than traditional mechanisms of conservative coalescing. Through pseudo-coalescing, rather than traditional coalescing via merging, OCC is able to identify sets of affinity-related vertices to which it would ideally like the assign the same color, while preserving the chordal graph property and ensuring that a $k$-coloring is found. On a set of interference graphs from a set of multimedia and embedded benchmarks, OCC found the optimal coalescing solution in all cases, and ran $2.30 \times$ faster than iterated register coalescing.

\section{References}

1. Boissinot B, Hack S, Grund D, De Dinechin BD, Rastello F (2008) Fast liveness checking for SSA-form programs. In: Proceedings of the 2008 IEEE/ACM symposium on code generation and optimization, Boston, MA, USA, 6-9 April 2008 
2. Bouchez F (2005) Register allocation and spill complexity under SSA. MS thesis, Technical Report RR2005-33, ENS-Lyon, Lyon France, August 2005

3. Bouchez F, Darte A, Guillon C, Rastello F (2006) Register allocation: what does the NP-Completeness proof of Chaitin et al. really prove? Or revisiting register allocation: why and how? In: Proceedings of the 19th international workshop on languages and compilers for parallel computing, New Orleans, LA, USA, November 2006

4. Bouchez F, Darte A, Rastello F (2007) On the complexity of register coalescing. In: Proceedings of the international symposium on code generation and optimization, San Jose, CA, USA, March 2008, pp 102-114

5. Bouchez F, Darte A, Rastello F (2007) On the complexity of spill everywhere under SSA form. In: Proceedings of the ACM SIGPLAN/SIGBED conference on languages, compilers, and tools for embedded systems, San Diego, CA, USA, June 2007, pp 103-112

6. Briggs P, Cooper KD, Harvey TJ, Simpson LT (1998) Practical improvements to the construction and destruction of static single assignment form. Softw Pract Exp 28:859-881

7. Briggs P, Cooper KD, Torczon L (1994) Improvements to graph coloring register allocation. ACM Trans Program Lang Syst 16:428-455

8. Brisk P, Dabiri F, Jafari R, Sarrafzadeh M (2006) Optimal register sharing for high-level synthesis of SSA form programs. IEEE Trans Comput-Aided Des Integr Circuits Syst 25:772-779

9. Brisk P, Sarrafzadeh M (2007) Interference graphs for procedures in static single information form are interval graphs. In: Proceedings of the 10th international workshop on software and compilers for embedded systems, Nice, France, 20 April 2007, pp 101-110

10. Brisk P, Verma AK, Ienne P (2007) An optimistic and conservative register assignment heuristic for chordal graphs. In: Proceedings of the international conference on compilers, architecture, and synthesis for embedded systems, Salzburg, Austria, 30 September-3 October 2007, pp 209-217

11. Budimlic Z, Cooper KD, Harvey TJ, Kennedy K, Oberg TS, Reeves SW (2002) Fast copy coalescing and live range identification. In: Proceedings of the ACM SIGPLAN conference on programming language design and implementation, Berlin, Germany, 17-19 June 2002, pp 25-32

12. Chaitin GJ (1982) Register allocation and spilling via graph coloring. In: Proceedings of the 1982 SIGPLAN symposium on compiler construction, Boston, MA, USA, 23-25 June 1982, pp 98-105

13. Chaitin GJ, Auslander MA, Chandra AK, Cocke J, Hopkins ME, Markstein PW (1981) Register allocation via coloring. Comput Lang 6:47-57

14. Choi J-D, Cytron R, Ferrante J (1991) Automatic construction of sparse data flow evaluation graphs. In: Proceedings of the 18th ACM SIGPLAN/SIGACT symposium on principles of programming languages, Orlando, FL, USA, 21-23 January 1991, pp 55-66

15. Cooper KD, Torczon L (2003) Engineering a compiler. Morgan Kaufmann, Los Altos (now a subsidiary of Elsevier)

16. Cytron R, Ferrante J, Rosen BK, Wegman MN, Zadeck FK (1991) Efficiently computing static single assignment form and the control dependence graph. ACM Trans Program Lang Syst 13:451-490

17. Ershov AP (1962) Reduction of the problem of memory allocation in programming to the problem of coloring the vertices of graphs. Dokl Akad Nauk SSSR 142. English translation in Sov Math 3:163-165 (1962)

18. Fabri J (1979) Automatic storage optimization. In: Proceedings of the ACM SIGPLAN symposium on compiler construction, Denver, CO, USA, 6-10 August 1979, pp 83-91

19. Farach-Colton M, Liberatore V (2000) On local register allocation. J Algorithms 37:37-65

20. Gavril F (1972) Algorithms for minimum coloring, maximum clique, minimum covering by cliques, and maximum independent set of a chordal graph. SIAM J Comput 1:180-187

21. George L, Appel AW (1996) Iterated register coalescing. ACM Trans Program Lang Syst 18:300-324

22. Grund D, Hack S (2007) A fast cutting-plane algorithm for optimal coalescing. In: 16th international conference on compiler construction, Braga, Portugal, 26-30 March 2007, pp 111-125

23. Guthaus MR, Ringenberg JS, Ernst D, Austin TM, Mudge T, Brown RB (2001) MiBench: a free, commercially representative embedded benchmark suite. In: Proceedings of the 4th IEEE workshop on workload characterization, Austin TX, USA, 2 December 2001, pp 3-14

24. Hack S, Goos G (2006) Register allocation for SSA-form programs in polynomial time. Inf Process Lett 98:150-155

25. Hailperin M (2005) Comparing conservative coalescing criteria. ACM Trans Program Lang Syst 27:571582

26. Hashimoto A, Stevens J (1971) Wire routing by optimizing channel assignment within large apertures. In: Proceedings of the 8th workshop on design automation, Atlantic City, NJ, USA, 28-30 June 1971, pp 155-169

27. Kaluskar V (2003) An aggressive live range splitting and coalescing framework for efficient register allocation. MS thesis, Georgia Institute of Technology, Atlanta, GA, USA, December 2003 
28. Kempe AB (1879) The geographical problem of the four colors. Am J Math 2:193-200

29. Lavrov SS (1961) Store economy in closed operator schemes. J Comput Math Math Phys 1:678-701. English translation in USSR Comput Math Math Phys 3:810-828 (1962)

30. Lee C, Potkonjak M, Mangione-Smith WH (1997) MediaBench: a tool for evaluating and synthesizing multimedia and communications systems. In: Proceedings of the 30th international symposium on microarchitecture, Research Triangle Park, NC, USA, 1-3 December 1997, pp 330-335

31. Lee JK, Palsberg J, Pereira FMQ (2007) Aliased register allocation for straight-line programs is NPComplete. In: Proceedings of the 34th international colloquium on automata, languages, and programming, Wroclaw, Poland, 9-13 July 2007, pp 680-691

32. Park J, Moon S-M (2004) Optimistic register coalescing. ACM Trans Program Lang Syst 26:735-765

33. Pereira FMQ, Palsberg J (2006) Register allocation after classical SSA elimination is NP-Complete. In: Proceedings of the 9th international conference on foundations of software science and computation structures, Vienna, Austria, 25-31 March 2006, pp 79-83

34. Poletto M, Sarkar V (1999) Linear scan register allocation. ACM Trans Program Lang Syst 21:895-913

35. Rastello F, De Ferriere F, Guillon C (2004) Optimizing translation out of SSA using renaming constraints. In: Proceedings of the 2nd IEEE/ACM symposium on code generation and optimization, San Jose, CA, USA, 20-24 March 2004, pp 265-278

36. Seward HH (1954) Information sorting in the application of electronic digital computers to business operations. MS thesis. Massachusetts Institute of Technology, Cambridge, MA, USA

37. Sreedhar VC, Ju RD-C, Gillies DM, Santhanam V (1999) Translating out of static single assignment form. In: Proceedings of the 6th international symposium on static analysis, 22-24 September 1999, pp 194-210

38. Tarjan RE, Yannakakis M (1984) Simple linear time algorithms to test chordality of graphs, test acyclicity of hypergraphs, and selectively reduce acylic hypergraphs. SIAM J Comput 13:566-579

39. Tseng C-J, Siewiorek DP (1986) Automated synthesis of data paths in digital systems. IEEE Trans Comput-Aided Des Integr Circuits Syst 5:379-395

40. Vegdahl SR (1999) Using node merging to enhance graph coloring. In: Proceedings of the ACM SIGPLAN conference on programming language design and implementation, Atlanta, GA, USA, 1-4 May 1999 , pp 150-154 